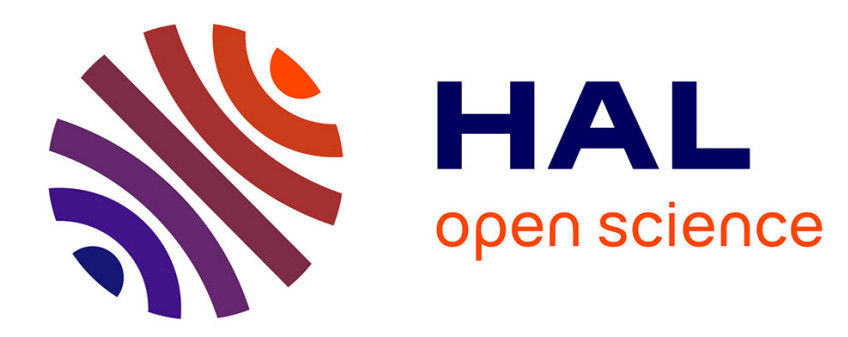

\title{
Phenomenological coefficients in a dilute BCC alloy for the dumbbell mechanism
}

Vincent Barbe, Maylise Nastar

\section{To cite this version:}

Vincent Barbe, Maylise Nastar. Phenomenological coefficients in a dilute BCC alloy for the dumbbell mechanism. Philosophical Magazine, 2007, 87 (11), pp.1649-1669. 10.1080/14786430600917223 . hal-00513747

\section{HAL Id: hal-00513747 \\ https://hal.science/hal-00513747}

Submitted on 1 Sep 2010

HAL is a multi-disciplinary open access archive for the deposit and dissemination of scientific research documents, whether they are published or not. The documents may come from teaching and research institutions in France or abroad, or from public or private research centers.
L'archive ouverte pluridisciplinaire HAL, est destinée au dépôt et à la diffusion de documents scientifiques de niveau recherche, publiés ou non, émanant des établissements d'enseignement et de recherche français ou étrangers, des laboratoires publics ou privés. 


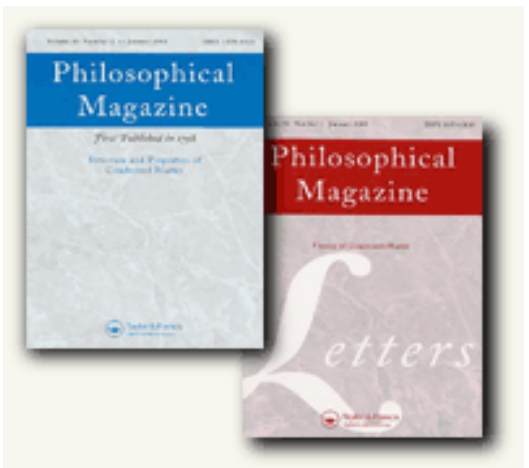

\section{Phenomenological coefficients in a dilute BCC alloy for the dumbbell mechanism}

\begin{tabular}{|c|c|}
\hline Journal: & Philosophical Magazine \& Philosophical Magazine Letters \\
\hline Manuscript ID: & TPHM-06-Apr-0101.R1 \\
\hline Journal Selection: & Philosophical Magazine \\
\hline $\begin{array}{l}\text { Date Submitted by the } \\
\text { Author: }\end{array}$ & 02-Jun-2006 \\
\hline Complete List of Authors: & $\begin{array}{l}\text { Barbe, Vincent; CEA Saclay, DMN/SRMP } \\
\text { Nastar, Maylise; CEA-Saclay, DMN/SRMP }\end{array}$ \\
\hline Keywords: & $\begin{array}{l}\text { alloys, atomic defects, atomic transport, atomistic simulation, } \\
\text { diffusion, irradiation effects, modelling, Monte-Carlo }\end{array}$ \\
\hline Keywords (user supplied): & dumbbell, dilute alloy, correlation effects \\
\hline \multicolumn{2}{|c|}{$\begin{array}{l}\text { Note: The following files were submitted by the author for peer review, but cannot be converted } \\
\text { to PDF. You must view these files (e.g. movies) online. }\end{array}$} \\
\hline \multicolumn{2}{|c|}{$\begin{array}{l}\text { Barbe main 0101.R1 19.07.tex } \\
\text { Barbe text 0101.R1 } 19.07 . \text { tex } \\
\text { Barbe barbib 0101.R1 19.07.bib } \\
\text { Barbe figures } 0101 . \mathrm{R} 1 \text { 19.07.tex }\end{array}$} \\
\hline
\end{tabular}

\section{ScholaronE \\ Manuscript Central}




\section{Abstract}

The Self-Consistent Mean Field Method is applied to calculate the transport coefficients in a dilute BCC alloy with the dumbbell diffusion mechanism. A first degree of approximation (first shell) of the SCMF formalism coincides with the formerly derived pair association method, and a second degree of approximation (second shell) leads to a more accurate analytical formulation. The SCMF results are compared with other formalisms as well as existing and new Monte Carlo simulations, including a solute-dumbbell binding energy. This theory shows a good balance between accuracy and maniability in the investigated systems, and a simple criterium is proposed for the preferential use of the first and second shell approximations.

Short title : Dumbbell in a dilute BCC alloy

\section{Introduction}

Within the framework of the linear thermodynamics of irreversible processes, the transport coefficients $L_{i j}$ are defined as the linear coefficients relating the flux of a species $i$ to the thermodynamic force applied on the species $j$. Theoretical calculations of those coefficients in model alloys have rised a considerable interest in the past decades, as their knowledge provides with information on the

\footnotetext{
*Author for correspondence. Email : maylise.nastar@cea.fr.
} 
coupling between fluxes of atoms and defects (e.g. a flux of solute atoms induced by a defect flux). Defect fluxes are of a particular importance in irradiated materials, where supersaturated vacancies and interstitials are permanently created and eliminate at defect sinks. As a consequence, attention has been specially paid to both main types of defects, vacancies and interstitials in the dumbbell configuration (two atoms on a single atomic site).

In a dilute alloy (say $B$ in the solvent $A$ ), very satisfying results have been achieved over the years for the vacancy mechanism [1]. However, the more complex dumbbell mechanism has not been given a complete treatment yet. In the FCC structure, Bocquet [2] calculated the transport coefficients for an energetic description of the system neglecting the interactions of the defect and the solute with neighbouring atoms, followed by Chaturvedi and Allnatt [3] and Singh and Chaturvedi [4]. A more complex calculation, including solute-dumbbell interaction, was proposed by Barbu [5] and completed by Allnatt et al. [6] and Okamura and Allnatt [7], all assuming $\langle 100\rangle$ dumbbells. In the BCC structure, Bocquet [8,9] calculated the diffusion coefficients of the solute and defect for the $\langle 110\rangle$ and $\langle 111\rangle$ orientations, but did not derive the entire set of transport coefficients. The first expression was due to Barbu and Lidiard [10] using the pair association method with the $\langle 110\rangle$ orientation : the results were found to be very simple, due to a low level of approximation, neglecting for instance the solute-dumbbell interactions. Recently, Sharma et al. [11] proposed a more systematic kinetic treatment for the same energetic description, based on the same formalism as Chaturvedi and Allnatt [3], hereafter refered to as the Chaturvedi formalism. However, due to the particular complexity of the jump mechanism for the $\langle 110\rangle$ dumbbell, the entire procedure was presented only for the translation mechanism. They nevertheless provided with the first Monte Carlo results for the dumbbell mechanism in a dilute alloy, which gives an insight of the accuracy of the different theories.

In this paper we address the calculation of the transport coefficients using the self-consistent http://mc.manuscriptcentral.com/pm-pml 
mean field theory (SCMF), initially proposed by Nastar et al. [12] for the vacancy mechanism (see also $[13,14])$, and newly adapted to the dumbbell mechanism $[15,16]$. This formalism has proved its efficiency by proposing the first expression of the transport coefficients in a concentrated BCC alloy for the dumbbell mechanism. We now explore the dilute limit of this alloy. Due to the relative simplicity of the expressions, we will take into account also in the present paper a complete thermodynamic description of the system, including dumbbell-substitutionnal interactions as well as interactions between the solute and solvent atoms.

Section 2 is devoted to the presentation of the atomic model and Section 3 to the the derivation of the transport coefficients. The latter is exactly the same as presented in reference [15], simply reduced to the dilute limit, so that we will only introduce the main equations in the present paper. As for the concentrated case, two approximations of the SCMF results are presented, denoted as first shell and second shell approximations, the corresponding results being summarized in section 4. Section 5 is shortly devoted to the Monte Carlo simulation technique, and the different approximations are discussed in Section 6, by comparison with existing formalisms (by Barbu and Lidiard or Sharma and Chaturvedi) on the basis of available and new Monte Carlo simulations.

\section{Atomic model}

As mentioned above, the stable geometry of the dumbbell that we consider in this study is the $\langle 110\rangle$ orientation. It is noted $A B_{\alpha}$, where $\alpha$ is one of the six possible orientations if both atoms are of the same species, and one of the twelve directions if else. In this structure the defect can experience four types of displacements, as presented by Bocquet [8]. The first one is an on-site rotation of an angle of $60^{\circ}$ toward another $\langle 110\rangle$ orientation, and the other three consist in the jump of one atom of the defect toward a substitutional atom to form a new defect, while the second atom of the initial defect 
remains in substitutional position. In the general case, one can write :

$$
A B_{\alpha}+C \rightarrow A+B C_{\beta}
$$

For the translation mechanism (T), $C$ is a 'target' nearest neighbour of $A B_{\alpha}$ and $\alpha=\beta$. The rotation-translation mechanism (RT) combines a translation plus a $60^{\circ}$ on-site rotation of the dumbbell $B C$. Eventually, another rotation-translation mechanism (RT2) combines a jump toward a second-neighbour site and a $90^{\circ}$ on-site rotation. This mechanism is temporarily ignored by the present version of the SCMF formalism for the dumbbell mechanism, which is restricted to a nearestneighbour (nn) description.

For simplicity we note $A B_{\|} C$ the configuration in which $C$ is on a target site for the $B$ atom of the dumbbell, $B A_{\|} C$ if $C$ is on a target site for the $A$ atom and $A B_{\perp} C$ if $C$ is on a non-target nn site of the dumbbell. Target sites as well as jump mechanisms are schemed on Figure 1.

\section{[ Insert Figure 1 about here ]}

The most common expression of the jump frequency for Equation (1) is $w_{A B / C}^{\alpha \beta}$, assuming that the frequency depends only on the nature of the three involved atoms and on the jump mechanism. This notation, due to Bocquet [2], was widely employed, particularly by Sharma et al. [11] in the dilute BCC alloy, but fails to describe a dumbbell-solute interaction. However, in our sense, it is necessary to treat the case of a dilute alloy more consistently. If we restrict to nn solute-dumbbell interactions, there is still a limited set of configurations to consider, and a limited set of jump frequencies relating the different configurations : we shall note $w_{i}(i=1,2 \ldots)$ each different jump frequency. This model is an adaptation to the BCC structure of the one proposed by Barbu [5], and an extension of the four-frequency model by Barbu and Lidiard [10] in the BCC dilute alloy, the latter neglecting atom-solute interactions. Table 1 describes each jump frequency $w_{i}$ and details the correspondence with the four-frequency model in absence of interactions. 


$$
\begin{aligned}
6 c_{A A}+12 c_{A B}+24 c_{A A_{\|} B}+24 c_{A A_{\perp} B} & =c_{I} \\
c_{B}^{\prime}+12 c_{A B}+24 c_{A A_{\|} B}+24 c_{A A_{\perp} B} & =c_{B},
\end{aligned}
$$

where $c_{I}$ and $c_{B}$ are the total concentrations of the system in dumbbells and solute atoms respectively, and $c_{B}^{\prime}$ is the concentration of free solute atoms (further than a nn distance from any dumbbell).

Those concentrations are calculated by means of the detailed balance in Appendix A, where a description of the jump frequencies in terms of interaction energies is also to be found. Although http://mc.manuscriptcentral.com/pm-pml 
such a description will be useful for the application to real alloys, we find more practical to derive the correlation coefficients in terms of the jump frequencies themselves.

The last step is to define jump probabilities $W$ as the product of a jump frequency $w$ by the concentration $c$ associated to its initial configuration :

$$
\begin{aligned}
W_{0} & =c_{A A} w_{0} \\
W_{1} & =c_{A B} w_{1} \\
W_{4} & =c_{A A_{\|} B} w_{4} \\
W_{6} & =c_{A A_{\perp} B} w_{6} \\
W_{R 4} & =c_{A A_{\|} B} w_{R 4}
\end{aligned}
$$

Within this framework, the detailed balance is very simply expressed as :

$$
\begin{aligned}
W_{2} & =W_{3} \\
W_{4} & =c_{B}^{\prime} W_{5} \\
W_{6} & =c_{B}^{\prime} W_{7} \\
W_{R 4} & =W_{R 5} .
\end{aligned}
$$

We can eventually define a mean jump occurence of an atom $A$ from one site to a given nn site :

$$
\begin{aligned}
W_{A}= & 3\left(2+\tau_{0}\right) W_{0}+3\left(2+\tau_{2}\right) W_{2}+9\left(2+\tau_{4}\right) W_{4}+9\left(2+\tau_{5}\right) c_{B}^{\prime} W_{5} \\
& +12\left(2+\tau_{6}\right) W_{6}+12\left(2+\tau_{7}\right) c_{B}^{\prime} W_{7} \\
= & 3\left(2+\tau_{0}\right) W_{0}+3\left(2+\tau_{2}\right) W_{2}+18\left(2+\tau_{5}\right) c_{B}^{\prime} W_{5}+24\left(2+\tau_{7}\right) c_{B}^{\prime} W_{7} .
\end{aligned}
$$

\section{The SCMF theory : derivation of the transport coeffi- cients}

In the SCMF theory [15], the transport coefficients are calcultated using a non-equilibrium distribution function of a system submitted to an homogeneous and vanishingly low gradient of chemical http://mc.manuscriptcentral.com/pm-pml 
potential. In this section, we present a shortened summary of this theory applied to the dumbbell mechanism in the BCC structure, to give to the reader the necessary material to perform the calculations.

\subsection{Non-equilibrium description}

Each configuration $\mathbf{n}$ of the system is described by the occupation numbers $n_{i}^{A}, \ldots, n_{i}^{A B_{\alpha}}, \ldots$, where $n_{i}^{A}$ is 1 if the site $i$ is occupied by the chemical species $A$ in substitutional position and 0 if else, and $n_{i}^{A B_{\alpha}}$ is 1 if the site $i$ is occupied by a dumbbell $A B$ with the orientation $\alpha, 0$ if else. Thus, the total occupations of site $i$ by the species $A$ or by a dumbbell are respectively :

$$
\begin{aligned}
N_{i}^{A} & =n_{i}^{A}+2 \sum_{\alpha} n_{i}^{A A_{\alpha}}+\sum_{\alpha, B \neq A} n_{i}^{A B_{\alpha}} \\
N_{i}^{I} & =\sum_{A, B, \alpha} n_{i}^{A B_{\alpha}} .
\end{aligned}
$$

Note that the sums over the orientations $\alpha$ contain six values for a dumbbell $A A$ and twelve for a dumbbell $A B$.

The equilibrium distribution function has the well-known form :

$$
P_{0}(\mathbf{n})=\exp \left[\beta G_{0}(\mathbf{n})\right]
$$

where $\beta=1 / k_{B} T$ is the inverse temperature and $G$ the Gibbs energy :

$$
G_{0}(\mathbf{n})=\Omega+\sum_{i, A} N_{i}^{A} \mu_{A}+\sum_{i} N_{i}^{I} \mu_{I}-H
$$

$\mu_{A}$ being the chemical potential of the species $A, \Omega$ a normalisation constant and $H$ the Hamiltonian of interactions between atoms and/or defects (see Appendix A).

Under an homogeneous gradient of chemical potential, the SCMF assumes a non-equilibrium distribution function of the form :

$$
\underset{\text { http://mc.manuscriptcentral.com/pm-pml }}{P}(\mathbf{n})=\exp \left[\beta\left(G_{0}(\mathbf{n})+\delta G\right)\right]
$$


where the correction $\delta G$ to the Gibbs energy is :

$$
\delta G(\mathbf{n})=\delta \Omega+\sum_{i, A} N_{i}^{A} \delta \mu_{i}^{A}+\sum_{i} N_{i}^{I} \delta \mu_{i}^{I}-h .
$$

Here $\delta \Omega$ is the correction to the normalization constant and $\delta \mu_{i}^{A}$ is the contribution of the gradient of chemical potential : unlike the equilibrium case, $\delta \mu_{i}^{A}$ depends on the site $i$.

The function $h$ is the result of the dynamic correlations between the occupation numbers of different sites under the influence of a gradient of chemical potential. Following Vaks [17], this function is a priori unknown and is supposed to take the most general form of an Hamiltonian, hence its name of effective Hamiltonian. It is composed of effective interactions $v$, which have nothing in common with thermodynamic interactions but their name and the alphabetic letter. In particular, the effective interactions are proportional to the gradients of chemical potential and are consequently directive, i.e. they obey the anti-symmetry relation :

$$
v_{i j}^{B A}=-v_{i j}^{A B}
$$

and more generally an effective interaction changes its sign by inverting the position of the concerned atoms. One consequence is that the effective interactions $v_{i j}^{A A}$ are always equal to zero.

A detailed presentation of the effective interactions and the antisymmetry property is to be found in [15] for a general multi-component concentrated alloy, with effective interactions limited to a nn range. In this study, we restrict ourselves to the common treatment of a dilute alloy and discard all configurations involving more than one solute atom. As a consequence, only six configurations are compatible with a nn description :

- $B$ belongs to the dumbbell ;

- $B$ is on a target site of the $A A$ dumbbell ;

- $B$ is on a nn non-target site of the $A A$ dumbbell. 
Each configuration is counted twice since the dumbbell can be oriented perpendicularly to the diffusion direction (superscript $I^{0}$ ) or toward the diffusion direction (superscript $I^{+}$). It is unnecessary to take into account the backward orientation, because of the antisymmetry property of the effective interactions. Figure 2 schemes those configurations and the associated effective interactions if the diffusion direction is assumed to be the $\langle 100\rangle$ direction. Note that the configuration $A B^{0}$ is invariant by symmetry and consequently gives rise to no effective interaction, so that the simple notation $v_{A B}$ is attributed to the configuration $A B^{+}$.

\section{[ Insert Figure 2 about here ]}

To conclude, recalling that the gradient of chemical potential is vanishingly low, so must be the correction $\delta G$, so that the non-equilibrium distribution function can be linearized with respect to the non-equilibrium terms. Using Equations (21), (23) and (24), one eventually obtains :

$$
P(\mathbf{n})=P_{0}(\mathbf{n})\left[1+\beta\left(\delta \Omega+\sum_{i, A} N_{i}^{A} \delta \mu_{i}^{A}+\sum_{i} N_{i}^{I} \delta \mu_{i}^{I}-h\right)\right]
$$

Generally, all non-equilibrium values may be time-dependent, and one could write $P(\mathbf{n}, t), \delta \Omega(t)$, $h(t) \ldots$ However, as we will see in the next section, the calculation is based on a steady-state description of a system out of equilibrium, so that the time dependence is unnecessary.

\subsection{Flux and kinetic equations}

The above non-equilibrium description is related to the transport coefficients of the system by a Master Equation, as introduced e.g. by Vaks [17] :

$$
\frac{d P(\mathbf{n}, t)}{d t}=\sum_{\tilde{\mathbf{n}}}[W(\tilde{\mathbf{n}} \rightarrow \mathbf{n}) P(\tilde{\mathbf{n}}, t)-W(\mathbf{n} \rightarrow \tilde{\mathbf{n}}) P(\mathbf{n}, t)],
$$

where $W(\tilde{\mathbf{n}} \rightarrow \mathbf{n})$ is the transition probability of a system in configuration $\tilde{\mathbf{n}}$ to the configuration $\mathbf{n}$ per time unit, and $P(\tilde{\mathbf{n}}, t)$ is the above defined non-equilibrium probability of the configuration $\tilde{\mathbf{n}}$. 
As a part of the distribution function, the effective interactions appear in the expression of the atomic fluxes between two neighbouring sites. Following the very same procedure as in Ref. [15], we express those fluxes in a dilute alloy as :

$$
\begin{aligned}
J_{i \rightarrow s}^{B}= & -3\left(2+\tau_{1}\right) W_{1}\left(\mu_{s}^{B}-\mu_{i}^{B}\right)-4\left(2+\tau_{1}\right) W_{1} v_{A B} \\
J_{i \rightarrow s}^{A}= & -W_{A}\left(\mu_{s}^{A}-\mu_{i}^{A}\right) \\
& +4\left(2+\tau_{2}\right) W_{2} v_{A B}+2\left(\left(2+\tau_{4}\right) W_{4}-\left(2+\tau_{2}\right) W_{2}\right)\left(2 v_{A A_{\|}^{+} B}+v_{A A_{\|}^{0} B}\right) .
\end{aligned}
$$

To this point, the effective interactions must be self-consistently calculated to give the expression of the transport coefficients. In the SCMF theory, they are determined by the use of kinetic equations, i.e. the corresponding moments of the distribution function $\left\langle n_{i}^{A B}\right\rangle,\left\langle n_{i}^{A A} n_{j}^{B}\right\rangle$ must be conserved. The kinetic equations are obtained by the same calculations as in Ref. [15], and are detailed below :

$$
\begin{aligned}
& \frac{d\left\langle n_{i}^{A B}\right\rangle}{d t}=2\left(2+\tau_{1}\right) W_{1}\left(\mu_{j+}^{B}-\mu_{i}^{B}\right)-2\left(2+\tau_{2}\right) W_{2}\left(\mu_{j+}^{A}-\mu_{i}^{A}\right) \\
& +\left[2\left(2+\tau_{2}\right) W_{2}+2\left(3+2 \tau_{1}\right) W_{1}+2 W_{R 1}\right] v_{A B} \\
& -2\left(1+\tau_{3}\right) W_{3} v_{A A_{\|}^{+} B}-2 W_{3} v_{A A_{\|}^{0} B} \\
& \frac{d\left\langle n_{i j}^{A A_{\|}^{+} B}\right\rangle}{d t}=\left[\left(2+\tau_{3}\right) W_{3}-\left(2+\tau_{4}\right) W_{4}\right]\left(\mu_{j+}^{A}-\mu_{i}^{A}\right) \\
& -\left(1+\tau_{2}\right) W_{2} v_{A B}+\left[3\left(2+\tau_{4}\right) W_{4}+\left(2+\tau_{3}\right) W_{3}+2 W_{R \|}+2 W_{R 5}\right] v_{A A_{\|}^{+} B} \\
& -W_{R \|} v_{A A_{\|}^{+} B}-W_{R \|} v_{A A_{\|}^{0} B}-W_{R 5} v_{A A_{\perp}^{+} B}-W_{R 5} v_{A A_{\perp}^{0} B} \\
& \frac{d\left\langle n_{i j}^{A A_{\|}^{0} B}\right\rangle}{d t}=\left[\left(2+\tau_{3}\right) W_{3}-\left(2+\tau_{4}\right) W_{4}\right]\left(\mu_{j+}^{A}-\mu_{i}^{A}\right) \\
& -2 W_{2} v_{A B}+\left[3\left(2+\tau_{4}\right) W_{4}+\left(2+\tau_{3}\right) W_{3}+2 W_{R \|}+2 W_{R 5}\right] v_{A A_{\|}^{0} B} \\
& -2 W_{R \|} v_{A A_{\|}^{+} B}-2 W_{R 5} v_{A A_{\perp}^{+B}} \\
& \frac{d\left\langle n_{i j}^{A A_{\perp}^{+} B}\right\rangle}{d t}=\left[4\left(2+\tau_{6}\right) W_{6}+2 W_{R 5}+2 W_{R \perp}\right] v_{A A_{\perp}^{+} B} \\
& \begin{array}{l}
-W_{R 5} v_{A A_{A}^{+} B}-W_{R 5} v_{A A_{A}^{0} B}-W_{R \perp} v_{A A^{+} B}-W_{R \perp} v_{A A_{\perp}^{0} B} \\
\text { http://mc.manuscriptceintral.com/pm-pmn }
\end{array}
\end{aligned}
$$




$$
\begin{aligned}
\frac{d\left\langle n_{i j}^{A A_{\perp}^{0} B}\right\rangle}{d t}= & {\left[4\left(2+\tau_{6}\right) W_{6}+2 W_{R 5}+2 W_{R \perp}\right] v_{A A_{\perp}^{+} B} } \\
& -2 W_{R 5} v_{A A_{\|}^{+} B}-2 W_{R \perp} v_{A A_{\perp}^{+} B}
\end{aligned}
$$

Here the notation $n_{i j}^{A A_{\|}^{+} B}$ for example stands for a dumbbell $A A^{+}$on site $i$ and an atom $B$ on target site $j$. We enjoin the reader to refer to Ref. [15], particularly Appendix A therein, for the technical counting of the effective interactions. An important remark is the use in the above relations of the reduced chemical potential of the chemical species :

$$
\mu_{i}^{A} \equiv \mu_{i}^{A}+\mu_{i}^{I}
$$

which guarantees that all reduced chemical potential gradients are independent.

The last step consists in putting Equations (30-34) to zero. It is straightforward to assess that the resulting effective interactions are linear combinations of the gradients of chemical potentials. As a consequence, combining with Equations (28) and (29) will lead to the Onsager equation, and thus the definition of the transport coefficients.

\section{Results}

The SCMF theory as presented in this paper leads to three different approximations of the transport coefficients, depending on the number of effective interactions one might take into account.

The crudest approximation is the neglecting of all dynamic correlations, or the uncorrelated transport coefficients. They are obtained by setting to zero all effective interactions, which leads to the relations :

$$
\begin{aligned}
& \frac{4 k_{B} T}{n a^{2}} L_{A A}^{(0)}=W_{A} \\
& \frac{4 k_{B} T}{n a^{2}} L_{A B}^{(0)}=0 \\
& \frac{4 k_{B} T}{n a^{2}} L_{B B}^{(0)}=3\left(2+\tau_{1}\right) W_{1}, \\
& \text { http://mc.manuscriptcentral.com/pm-pml }
\end{aligned}
$$


where $n$ is the number of atoms per unit volume and $a$ is the lattice parameter.

The first shell approximation consists in setting the effective interactions involving three atoms to zero and then solve Equation (30) alone. The transport coefficients in the first shell approximation have the following expression :

$$
\begin{aligned}
\frac{4 k_{B} T}{n a^{2}} L_{A A}^{(1 s)} & =W_{A}-\frac{4\left(2+\tau_{2}\right)^{2} W_{2}^{2}}{\left(3+2 \tau_{1}\right) W_{1}+\left(2+\tau_{2}\right) W_{2}+W_{R 1}} \\
\frac{4 k_{B} T}{n a^{2}} L_{A B}^{(1 s)} & =\frac{4\left(2+\tau_{2}\right)^{2} W_{2} W_{1}}{\left(3+2 \tau_{1}\right) W_{1}+\left(2+\tau_{2}\right) W_{2}+W_{R 1}} \\
& =\frac{4 k_{B} T}{n a^{2}} L_{B A}^{(1 s)} \\
\frac{4 k_{B} T}{n a^{2}} L_{B B}^{(1 s)} & =\frac{\left(2+\tau_{1}\right) W_{1}\left(\left(1+2 \tau_{1}\right) W_{1}+3\left(2+\tau_{2}\right) W_{2}+3 W_{R 1}\right)}{\left(3+2 \tau_{1}\right) W_{1}+\left(2+\tau_{2}\right) W_{2}+W_{R 1}} .
\end{aligned}
$$

It must be noted that, though the first shell approximation neglects the kinetic correlations between the solute and the $A A$ dumbbell, it is fully compatible with a thermodynamic interaction between both species, which will actually act on the different jump frequencies.

In the second shell approximation, we calculate in principle the transport coefficients on the basis of all kinetic equations. However, if an analytic resolution of the set of five kinetic equations is always possible, the final expression is very lengthy and may not be of interest. Consequently we chose to derive the analytical calculation in the absence of on-site rotation : one notes that in this particular case, the effective interactions between a dumbbell and a solute atom on a non-target nn site are equal to zero (Equations (33) and (34)), so that the number of independent equations reduces to three. Still, the thermodynamic interaction between a dumbbell and a solute on a non-target nn site continues to play a role, as it controls the concentrations of the different configurations and the 
associated jump frequencies. The transport coefficients are :

$$
\begin{aligned}
\frac{4 k_{B} T}{n a^{2}} L_{A A}^{(2 s)} & =W_{A}-6 \frac{\left(\left(2+\tau_{3}\right) W_{3}-\left(2+\tau_{4}\right) W_{4}\right)^{2}}{\left(2+\tau_{3}\right) W_{3}+3\left(2+\tau_{4}\right) W_{4}}-\frac{8 \Psi^{2}\left(2+\tau_{4}\right) W_{4}\left(\left(2+\tau_{3}\right) W_{3}-\left(2+\tau_{4}\right) W_{4}\right)}{\left(3+2 \tau_{1}\right) W_{1}+\left(2+\tau_{2}-\theta \Psi\right) W_{2}} \\
\frac{4 k_{B} T}{n a^{2}} L_{A B}^{(2 s)} & =\frac{4\left(2+\tau_{2}\right)^{2} W_{1} \times\left(4 \Psi W_{4}\right)}{\left(3+2 \tau_{1}\right) W_{1}+\left(2+\tau_{2}-\theta \Psi\right) W_{2}} \\
& =\frac{4 k_{B} T}{n a^{2}} L_{B A}^{(2 s)} \\
\frac{4 k_{B} T}{n a^{2}} L_{B B}^{(2 s)} & =\frac{\left(2+\tau_{1}\right) W_{1}\left(\left(1+2 \tau_{1}\right) W_{1}+3\left(2+\tau_{2}-\theta \Psi\right) W_{2}\right)}{\left(3+2 \tau_{1}\right) W_{1}+\left(2+\tau_{2}-\theta \Psi\right) W_{2}}
\end{aligned}
$$

where we have introduced the quantities $\Psi$ and $\theta$ as :

$$
\begin{aligned}
\Psi & =\frac{\left(2+\tau_{3}\right) W_{3}}{3\left(2+\tau_{4}\right) W_{4}+\left(2+\tau_{3}\right) W_{3}} \\
\theta & =\frac{\left(1+\tau_{2}\right)^{2}+2}{1+\tau_{2}}
\end{aligned}
$$

\section{Monte Carlo simulations}

To test the key results of our and competing theories, we performed a series of Monte Carlo simulations. We used a simulation box of 512 atomic sites with periodic boundary conditions, containing only one dumbbell and one solute atom, so that $c_{I}=1 / 512$ and $c_{B}=1 / 513$ : in these conditions we respect the assumption of the dilute alloy model and never introduce any configuration involving more than one solute atom. A general description of the simulation was given by Murch [18].

The transport coefficients are calculated by the generalized Einstein relations [19] :

$$
\begin{aligned}
& k_{B} T V L_{A A}=\frac{\left\langle\Delta \mathbf{R}_{A}^{2}\right\rangle}{6 t} \\
& k_{B} T V L_{A B}=\frac{\left\langle\Delta \mathbf{R}_{A} \Delta \mathbf{R}_{B}\right\rangle}{6 t}
\end{aligned}
$$

where $\Delta \mathbf{R}_{A}$ is the total displacement of all atoms of species $A$ during the time $t$, and $V$ is the atomic volume. The averaging $\langle$.$\rangle is operated over at least 10^{5}$ observations : each observation contains in average at least five jumps per atom of each atomic species (i.e. 5 jumps of the $B$ atom and 2560 jumps of $A$ atoms), and we impose additionaly that each observation contains at least five jumps http://mc.manuscriptcentral.com/pm-pml 
of the type $A A / A$ to ensure that the defect doesn't remain "stuck" to the solute atom. Given the chosen jump frequency ratios which can outpass 1000:1, we might consider as satisfying the obtained relative error of $10 \%$ to $20 \%$ in the transport coefficients.

In the presented simulations, we use the value $\tau_{i}=1$ for all frequencies $w_{i}$. However, the accuracy of the different approximations of the SCMF theory seems unaffected by the coefficients $\tau_{i}$. Moreover, in order to use the analytical expressions derived for the second shell approximation, we will discard the on-site rotation.

\section{Discussion}

In this section the results of the SCMF theory are compared to the other available theories (by Sharma et al. [11] using the formalism of Chaturvedi, and by Barbu and Lidiard [10] using the pair association method) and to Monte Carlo simulations. A first part will be devoted to a theoretical comparison of all formalisms, including both approximations of the SCMF theory, and the second and third parts will concern the Monte Carlo simulations, respectively within and beyond the fourfrequency model. The effect of on-site rotation is then briefly discussed.

\subsection{An analytical comparison of the formalisms}

Before addressing the competing formalisms, we will focus on both approximations of the SCMF. As it is visible from the expressions of the transport coefficients, the main differences arise from the addition of factors $\Psi$ and $\theta$ (defined by Equations (39) and (40) in the second shell approximation). The physical meaning of $\Psi$ is clearly the competition between the possible jumps of a $A A$ defect with a neighbouring solute atom : the formation of a mixed dumbbell (frequency $W_{3}$ ) or a jump toward one of the three other target sites with the frequency $W_{4}$. As emphasized by the expressions of the transport coefficients, this contribution is the most important difference between the first shell and second shell approximations : we notice in particular the change in the factor $L_{A B}$, where the jump http://mc.manuscriptcentral.com/pm-pml 
probability $W_{2}$ (equal to $W_{3}$ by the detailed balance) is replaced by $\Psi W_{4}$ in the numerator. Hence, the first shell approximation should be sufficient as long as both values are not too different, i.e. the ratio $W_{3} / W_{4}=w_{3} / w_{4}$ is not too high.

On the contrary, the factor $\theta$ seems to be only a slight geometrical correction. Actually, as $\Psi$ is always lower than 1 , the correction $\theta \Psi$ cannot reach values above $3 / 2$ in absence of translation or 2 if $\tau_{2}=1$. This factor is the only difference between both approximations in the expression of $L_{B B}$, and the subsequent variation of $L_{B B}$ will not exceed $50 \%$.

To return to the first shell approximation, it is useful to notice that it is strictly equivalent to the Barbu and Lidiard formalism, provided that all $\tau_{i}$ factors be equal to 1 . Actually, the pair association method and the SCMF theory are both based on the resolution of the same kinetic equations, and this similarity could have been expected. From this point of view, it is most probable that a second shell extension of the work of Barbu and Lidiard would have led to the same results as ours, and that the second shell approximation of the SCMF in a dilute FCC alloy would equal the results of Allnatt et al. [6] using the pair association method. It can thus be concluded that the pair association method, presently restricted to the dilute alloys, is contained in the more general SCMF formalism.

On the other extremity, a comparison of the SCMF with Chaturvedi's formalism was already performed in Ref. [15] for the case of concentrated FCC alloys : it had been highlighted that the formalism of Chaturvedi could be understood as the SCMF formalism including infinite-range pairwise effective interactions. Chaturvedi's formalism is then expected to be more accurate than the present version of the SCMF theory, although the case of the concentrated FCC alloy pointed out that the possible gain in accuracy was balanced by a considerable degree of complexity. In the dilute BCC alloy, this complexity resulted in the impossibility to calculate the transport coefficients but for the mechanism of simple translation. In this particular case, an analytical formulation of the transport coefficients is provided (Equations (45), (48) and (49) of Ref. [11]). Those equations contain a factor http://mc.manuscriptcentral.com/pm-pml 
$Q$ (Equation (46) in Ref. [11]) similar in its spirit to the factor $\Psi$ introduced by the SCMF theory. In conclusion, and before any discussion on the results themselves, this SCMF application appears like an intermediate approximation between the simple first shell pair association method and the more systematic formalism of Chaturvedi, the latter being available only for the translation mechanism.

\section{$6.2 \quad$ Four-frequency model}

In order to compare the accuracy of all models, we first restrict ourselves to the simple translation mechanism. Figure 3 shows the results of all three approximations for the following set of jump frequencies : $w_{A A / A}=0.01, w_{A A / B}=0.05, w_{A B / A}=1, w_{B A / A}=0.1$, as a function of the solute concentration, in terms of the correlation factor :

$$
f_{A B}^{(A)}=\frac{L_{A B}}{L_{A A}^{(0)}} .
$$

The figure also contains Monte Carlo simulations by Sharma et al. [11], in which the jump frequencies $w_{B B / A}=1, w_{B A / B}=0.15, w_{A B / B}=1, w_{B B / B}=1$ are included, i.e. the simulation box contains several solute atoms, and configurations involving more than one solute atom actually appear. To avoid the possible confusion due to the scattering, we represent only the values of the coefficient $f_{A B}^{(A)}$. The differences between the three models are equivalent for $f_{A B}^{(B)}$, and negligible for $f_{A A}$ and $f_{B B}$. The SCMF results in both approximations are calculated for the translation mechanism only, by setting to zero all terms which are not multiplied by a factor $\tau_{i}$ : as a consequence, the first shell results are different from those predicted by Barbu and Lidiard [10], who did not differentiate the translation and RT mechanisms.

\section{[ Insert Figure 3 about here ]}

Two main results are to be seen from Figure 3. On the first hand, the second shell approximation seems to be surprisingly more accurate than Chaturvedi's formalism. This result was unexpected 
as the latter contains information about infinite-range correlations, and should be superior. The difference observed with respect to the Monte Carlo simulations could arise from the configurations containing more than one solute atom : those configurations are taken into account by the Monte Carlo simulations, and not by the theoretical models. Nevertheless, it is also to notice that the difference between the second-shell approximation and the more complex formalism of Chaturvedi is not essential and reduces to a factor 1.5, so that the nearest-neighbour kinetic treatment of the SCMF might be sufficient in most cases of interest.

The second information is that the concentration of solute may not be a key parameter for the comparison of the different formalisms : hence, one observes that the difference between all formalisms remains practically constant in the entire concentration range. The reason is that all calculations are made to the first order with respect to $c_{B}$ and will lead to linear expressions in $c_{B}$, so that the only differences lie in the coefficients of the linear expressions.

To answer those points, we now focus on new Monte Carlo measurements in a simulation box containing only one solute atom, and choose to keep a fixed concentration for varying jump frequency ratios. As we have seen in the first part of the discussion, the main difference between first and second shell concerns the treatment of the ratio $w_{3} / w_{0}$ (since in the four-frequency model $w_{4}$ is replaced by $w_{0}$, see Table 1$)$. Consequently, we keep all three frequencies $\left\{w_{0}, w_{1}, w_{2}\right\}$ equal to 1 and let $w_{3}$ vary from $0.1(\Psi=0.033)$ to $10^{5}(\Psi=1)$. We further assume that the jump frequencies for the simple translation and rotation-translation are equal $\left(\tau_{i}=1\right.$ for all $\left.i\right)$, which is more realistic but discards the formalism of Sharma et al., and we neglect for simplicity the on-site rotation mechanism. The resulting transport coefficients are to be seen on Figure 4.

\section{[ Insert Figure 4 about here ]}

In this particular set of jump frequencies, the first shell approximation of the SCMF (or the

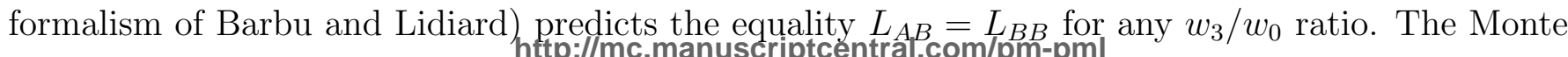


Carlo simulations show that it is generally not the case, and that the first shell approximation can overestimate the cross coefficient $L_{A B}$ as well as the coefficient $L_{A A}$ by several orders of magnitude for large $w_{3} / w_{0}$ ratios. On the contrary, the second shell approximation is surprisingly accurate in this domain. Nevertheless, we must notice that the first shell approximation is sufficient for a $w_{3} / w_{0}$ ratio between 0 and 10, which supports the conclusions reached in the preceeding section from a simple observation of the analytiical expressions. A more systematic study involving the variation of frequencies $w_{0}, w_{1}$ and $w_{2}$ shows that the first shell approximation is actually satisfying in all cases but for a high $w_{3} / w_{0}$ ratio.

The discrepancy for the factor $L_{A A}$ in the case of large jump frequency ratios is attributed to a very poor accuracy of the Monte Carlo simulations in this domain : as the association frequency $w_{3}$ is much larger than the escape frequency $w_{0}$, the dumbbell will stay in the neighbourhood of the solute atom, and the collective movement of the solvent atoms are not satisfyingly explored.

\subsection{Beyond the four-frequency model}

The relative simplicity of the SCMF formalism allowed to introduce an energetic description of the system beyond the four-frequency model, including particularly solute-dumbbell nearest-neighbour interactions. We hereby use this possibility to investigate a "stairs-like" behaviour of the energetic description. This behaviour is sketched in Figure 5. The mixed dumbbell is given a fixed binding energy $\epsilon_{A B}$, the dumbbell $A A$ is assumed to have no binding energy, and the solute-dumbbell complex $A A_{\|} B$ ( $B$ on a target site of $\left.A A\right)$ has an intermediate binding energy $V_{A A_{\|} B}$ between zero and $\epsilon_{A B}$. In this case, the complete dissociation of the mixed dumbbell will occur in two consecutive steps, with frequencies $w_{2}$ and $w_{4}$.

\section{[ Insert Figure 5 about here ]}


Figure 6 shows the corresponding transport coefficients as predicted by both approximations of the SCMF theory, compared with Monte Carlo simulations under the assumption that all factors $\tau_{i}$ are equal to 1 . The first shell approximation is already very satisfying for diagonal factors $L_{A A}$ and $L_{B B}$, whereas the second approximation is necessary for a good prediction of the cross-coefficient $L_{A B}$ when the solute-dumbbell interaction $V_{A A_{\|} B}$ is not zero. Such a result was actually to expect if one observes the 'return probability' $\Psi$ as defined by Equation (39). In our particular case, this probability takes the value :

$$
\Psi \equiv \frac{w_{3}}{w_{3}+3 w_{4}}=\frac{1}{1+3 \exp \left(\beta V_{A A_{\|} B}\right)}
$$

If $V_{A A_{\|} B}$ approaches the value of $\epsilon_{A B}$, the factor $\Psi$ is nearly equal to unity, which the first shell of the SCMF fails to account for.

\section{[ Insert Figure 6 about here ]}

Moreover, beyond the simple comparison between both approximations, one can observe on Figure 6 that all three transport coefficients are practically independent on the value of the solute-dumbbell interaction. This result supports a widely accepted hypothesis, which states that in presence of multiple energy barriers, only the total energy barrier needs to be taken into account for a satisfying modelling of the phenomena. However, if this modelling should be based on the more simple SCMF first shell approximation, the correct limit is the one which ignores the solute-dumbbell interaction. http://mc.manuscriptcentral.com/pm-pml 


\subsection{Effect of the on-site rotation}

The on-site rotation mechanism was discarded in the Monte Carlo simulations as well as the second shell expressions (Equations (38)) for complexity reasons. However, it deserves some rapid remarks.

On the one hand, the decorrelating effect of rotation already noticed by Barbu and Lidiard [10] is confirmed by the equivalent first shell approximation (see the presence of $W_{R 1}$ at the denominator of $L_{A B}$ in Equation (37)). This effect is relatively easy to understand : if the rotation frequency is high, a large number of rotations will occur between two consecutive jumps (we define a 'jump' by a change of the atomic site of the defect), so that all eight nn sites of the defect are potentially target sites. Consequently, the relative weight of the return frequency of the defect after one given jump, which is responsible for the correlation effects $[20,14]$, will considerably decrease, hence the lowering of the correlation effects. It must be noticed that the rotation frequency has no effect on the uncorrelated coefficients $L_{A A}^{(0)}$ and $L_{B B}^{(0)}$, which means that the rotation mechanism eventually leads to an acceleration of the diffusion.

On a quantitative point of view, the authors recently showed [16] that the first shell approximation generally overestimates the decorrelating effect of rotation in a concentrated BCC alloy. The first shell approximation remains quantitative when the rotation frequency is lower or equal to the slowest characteristic jump frequency, while the second shell numerical results were found on the contrary very close to the Monte Carlo simulations for any value of the rotation frequency. This result is valid upon the whole concentration range, including the dilute limit. 


\section{Conclusion}

We presented the application of the SCMF theory, recently adapted to the dumbbell mechanism [15], to the case of a dilute BCC alloy. A first shell approximation coincides with earlier results by Barbu and Lidiard [10], indicating that the pair association method may be contained within the SCMF formalism. A second shell approximation, which leads to usable analytical results in absence of onsite rotation, is found similar to a recent work by Sharma el al. [11], though the latter was much more complex in its construction, and limited to the simple translation mechanism.

Comparisons with Monte Carlo simulations involving or not solute-dumbbell interactions proved the general accuracy of the second shell approximation. However, the simpler first shell expresssion is still valid while the frequency ratio between an association jump AA/B and a competing AA/A jump $w_{3} / w_{0}$ is not greater than 10 . In presence of solute-dumbbell interactions, this ratio should only be replaced by $w_{3} / w_{4}$.

Given the identification observed between the SCMF theory and the pair association method, application of the SCMF theory to the FCC structure does not seem necessary, as detailed studies in the second shell approximation were already devoted to the subject [6]. Forthcoming work will then be preferentially focused on the generalization of the model of an interacting alloy to the concentrated domain.

\section{Acknowledgements}

The authors are grateful to J.L. Bocquet, A. Barbu, C. C. Fu and E. Meslin for stimulating discussions. Support by F. Soisson and E. Clouet for the MC simulations was also appreciated. Part of this work has been funded by the joint research program SMIRN between EDF, CNRS and CEA and by the European PERFECT project supported by the European Commission (FI60-CT-2003-508840). 


\section{Appendix A : concentrations of dumbbells in a dilute inter- acting alloy}

In this section, we calculate the probabilities of the different configurations appearing in a dilute BCC alloy with help of a full energetic description of the system. This description involves the binding energies of different types of dumbbells $\left(\epsilon_{A A}\right.$ and $\left.\epsilon_{A B}\right)$, interactions between nearest-neighbour substitutional atoms $\left(V_{A A}\right.$ and $\left.V_{A B}\right)$, as well as solute-dumbbell interactions when the solute is on a target site $\left(V_{A A_{\|} B}\right)$ or a nn non-target site $\left(V_{A A_{\perp} B}\right)$. The concentrations to calculate are $c_{A A}, c_{A B}$, $c_{A A_{\perp} B}, c_{A A_{\|} B}$ and $c_{B}^{\prime}$ as defined in Section 3. Note in particular that each concentration involves one only orientation and direction of the dumbbell and one only neighbour for $c_{A A_{\perp} B}$ and $c_{A A_{\|} B}$, hence the conservation properties of Equations (6) and (7). The principle is to solve the equations of the detailed balance under the conditions of both conservation equations.

We first express the frequency ratios involved in the detailed balance within the framework of interactions. Those ratios are obtained under the assumption of a classical bond-breaking model :

$$
\begin{aligned}
& \frac{w_{2}}{w_{3}}=\exp \left[\beta\left(-\epsilon_{A A_{\|} B}-\epsilon_{A A}-7 V_{A B}+\epsilon_{A B}+7 V_{A A}\right)\right] \\
& \frac{w_{4}}{w_{5}}=\exp \left[\beta\left(\epsilon_{A A_{\|} B}+V_{A A}-V_{A B}\right)\right] \\
& \frac{w_{6}}{w_{7}}=\exp \left[\beta\left(\epsilon_{A A_{\perp} B}+V_{A A}-V_{A B}\right)\right]
\end{aligned}
$$

The equations of the detailed balance (Equations (13) to (15)) can then be expressed as :

$$
\begin{aligned}
& \frac{c_{A A_{\|} B}}{c_{A B}}=\exp \left[\beta\left(-\epsilon_{A A_{\|} B}-\epsilon_{A A}-7 V_{A B}+\epsilon_{A B}+7 V_{A A}\right)\right] \\
& \frac{c_{A A_{\|} B}}{c_{A A} c_{B}}=\exp \left[-\beta\left(\epsilon_{A A_{\|} B}+V_{A A}-V_{A B}\right)\right] \\
& \frac{c_{A A_{\perp} B}}{c_{A A} c_{B}}=\exp \left[-\beta\left(\epsilon_{A A_{\perp} B}+V_{A A}-V_{A B}\right)\right] .
\end{aligned}
$$

It is then straightforward to derive the expression of the different concentrations. For simplicity, 
we introduce the different weights $P_{i}$ as :

$$
\begin{aligned}
P_{A B} & =\exp \left[-\beta\left(\epsilon_{A B}-\epsilon_{A A}+8\left(V_{A A}-V_{A B}\right)\right)\right] \\
P_{A A_{\|} B} & =\exp \left[-\beta\left(\epsilon_{A A_{\|} B}+V_{A A}-V_{A B}\right)\right] \\
P_{A A_{\perp} B} & =\exp \left[-\beta\left(\epsilon_{A A_{\perp} B}+V_{A A}-V_{A B}\right)\right]
\end{aligned}
$$

so that the final concentrations are simply expressed by :

$$
\begin{aligned}
c_{A A} & =\frac{1}{1+2 c_{B}^{\prime} P_{A B}+4 c_{B}^{\prime} P_{A A_{\|} B}+4 c_{B}^{\prime} P_{A A_{\perp} B}} \times \frac{c_{I}}{6} \\
c_{A B} & =\frac{c_{B}^{\prime} P_{A B}}{1+2 c_{B}^{\prime} P_{A B}+4 c_{B}^{\prime} P_{A A_{\|} B}+4 c_{B}^{\prime} P_{A A_{\perp} B}} \times \frac{c_{I}}{6} \\
c_{A A_{\|} B} & =\frac{c_{B}^{\prime} P_{A A_{\|} B}}{1+2 c_{B}^{\prime} P_{A B}+4 c_{B}^{\prime} P_{A A_{\|} B}+4 c_{B}^{\prime} P_{A A_{\perp} B}} \times \frac{c_{I}}{6} \\
c_{A A_{\perp} B} & =\frac{c_{B}^{\prime} P_{A A_{\perp} B}}{1+2 c_{B}^{\prime} P_{A B}+4 c_{B}^{\prime} P_{A A_{\|} B}+4 c_{B}^{\prime} P_{A A_{\perp} B}} \times \frac{c_{I}}{6} .
\end{aligned}
$$

However, to be consistent with the kinetic treatment of the alloy, which is processed to the first order in $c_{B}^{\prime}$, one must express the concentrations in the same limit :

$$
\begin{aligned}
c_{A A} & =\left(1-c_{B}^{\prime} P\right) \frac{c_{I}}{6} \\
c_{A B} & =c_{B}^{\prime} P_{A B} \frac{c_{I}}{6} \\
c_{A A_{\|} B} & =c_{B}^{\prime} P_{A A_{\|} B} \frac{c_{I}}{6} \\
c_{A A_{\perp} B} & =c_{B}^{\prime} P_{A A_{\perp} B} \frac{c_{I}}{6} .
\end{aligned}
$$

where we have introduced for simplicity the global weight :

$$
P=2 P_{A B}+4 P_{A A_{\|} B}+4 P_{A A_{\perp} B}
$$

At this point, we recall that the concentration $c_{B}^{\prime}$ is the concentration of free solute atoms in the system, i.e. solute atoms which are not involved in a mixed dumbbell nor in a solute-dumbbell complex. If $c_{B}$ is much higher than $c_{I}$, then we can consider that $c_{B}^{\prime}$ equals $c_{B}$, which corresponds http://mc.manuscriptcentral.com/pm-pml 
to the physical case of a normal alloy. However, in our Monte Carlo simulations, we assumed the equality $c_{I}=c_{B}$ to avoid configurations with several solute atoms. The exact value of $c_{B}^{\prime}$ is then given by solving Equation (7) :

$$
c_{B}^{\prime}=\frac{c_{B}}{1+c_{I} P} .
$$

http://mc.manuscriptcentral.com/pm-pml 


\section{References}

[1] A. R. Allnatt and A. B. Lidiard. Atomic Transport in Solids. Cambridge University Press, 1993.

[2] J.-L. Bocquet. Phil. Mag. A, 47:547, 1983.

[3] D. K. Chaturvedi and A. R. Allnatt. Phil. Mag. A, 65:1169, 1992.

[4] R. Singh and D. K. Chaturvedi. Phys. Rev. B, 48:16366, 1993.

[5] A. Barbu. Acta Met., 28:499, 1980.

[6] A. R. Allnatt, A. Barbu, A. D. Franklin, and A. B. Lidiard. Acta Met., 31:1307, 1983.

[7] Y. Okamura and A. R. Allnatt. Phil. Mag. A, 54:773, 1986.

[8] J.-L. Bocquet. Technical Report CEA-R-5503 (E), CEA, 1989.

[9] J.-L. Bocquet. Phil. Mag. A, 63:157, 1991.

[10] A. Barbu and A. B. Lidiard. Phil. Mag. A, 74:709, 1996.

[11] S. Sharma, D. K. Chaturvedi, I. V. Belova, and G. E. Murch. Phil. Mag. A, 81:431, 1993.

[12] M. Nastar, V. Yu. Dobretsov, and G. Martin. Phil. Mag. A, 80:155, 2000.

[13] M. Nastar. Phil. Mag., 85:3767, 2005.

[14] V. Barbe and M. Nastar. Phil. Mag., 86:1513, 2006.

[15] V. Barbe and M. Nastar. Phil. Mag., in press, 2006.

[16] M. Nastar and V. Barbe. Faraday Dicuss., (DOI:10.1039/ B605834E), 2006.

[17] V. G. Vaks, S. V. Beiden, and V. Yu. Dobretsov. JETP Lett., 61:68, 1995. http://mc.manuscriptcentral.com/pm-pml 
[18] G. E. Murch and A. S. Nowick. Diffusion in Crystalline Solids. Academic Press, Orlando, FL, 1982.

[19] A. R. Allnatt and E. L. Allnatt. Phil. Mag. A, 64:341, 1984.

[20] J. R. Manning. Diffusion Kinetics for Atoms in Cristals. Van Nostrand, Princeton, N. J., 1968. 
Table 1 : Jump and rotation frequencies in a dilute alloy considering interactions between a dumbbell and a substitutionnal nn solute atom. Jump frequencies refer to the rotation-translation mechanism only. Initial and final configurations are independent on the orientation relative to the $X$ axis, i.e. $A B$ is equivalent to $B A . A A$ stands for a configuration where the dumbbell $A A$ has no solute atom in nn substitutionnal position.

http://mc.manuscriptcentral.com/pm-pml 


\begin{tabular}{c|cccc}
\hline Frequency & $\begin{array}{c}\text { Initial } \\
\text { configuration }\end{array}$ & $\begin{array}{c}\text { Target } \\
\text { atom }\end{array}$ & $\begin{array}{c}\text { Final } \\
\text { configuration }\end{array}$ & $\begin{array}{c}\text { Four-frequency } \\
\text { model }\end{array}$ \\
\hline \hline$w_{0}$ & $A A$ & $A$ & $A A$ & $w_{0}$ \\
$w_{1}$ & $A B$ & $A$ & $B A$ & $w_{1}$ \\
$w_{2}$ & $B A$ & $A$ & $A A_{\|} B$ & $w_{2}$ \\
$w_{3}$ & $A A_{\|} B$ & $B$ & $A B$ & $w_{3}$ \\
$w_{4}$ & $A A_{\|} B$ & $A$ & $A A$ & $w_{0}$ \\
$w_{5}$ & $A A$ & $A$ & $A A_{\|} B$ & $w_{0}$ \\
$w_{6}$ & $A A_{\perp} B$ & $A$ & $A A$ & $w_{0}$ \\
$w_{7}$ & $A A$ & $A$ & $A A_{\perp} B$ & $w_{0}$ \\
$w_{R 0}$ & $A A$ & & $A A$ & $w_{R 0}$ \\
$w_{R 1}$ & $A B$ & & $A B$ & $w_{R 1}$ \\
$w_{R 4}$ & $A A_{\|} B$ & & $A A_{\perp} B$ & $w_{R 0}$ \\
$w_{R 5}$ & $A A_{\perp} B$ & & $A A_{\|} B$ & $w_{R 0}$ \\
$w_{R \|}$ & $A A_{\|} B$ & & $A A_{\|} B$ & $w_{R 0}$ \\
$w_{R \perp}$ & $A A_{\perp} B$ & & $A A_{\perp} B$ & $w_{R 0}$ \\
\hline
\end{tabular}

Table 1:

http://mc.manuscriptcentral.com/pm-pml 
Figure 1 : Jump mechanism for the $\langle 110\rangle$ dumbbell (in grey) in the BCC structure. Atoms in black are on target sites, white atoms are on nn non-target sites. (a) Simple translation mechanism ; (b)-(c) : rotation-translation mechanism.

Figure 2 : Geometry of the $\langle 110\rangle$ dumbbell in the BCC structure in the case of a one-dimensional diffusion. The configurations to be taken into account are (a): $A B^{0} ;(\mathrm{b}): A A_{\|}^{0} B ;(\mathrm{c})$ : $A A_{\perp}^{0} B ;(\mathrm{d}): A B^{+} ;(\mathrm{e}): A A_{\|}^{+} B ;(\mathrm{f}): A A_{\perp}^{+} B$. The arrow indicates the orientation of the diffusion fluxes.

Figure 3 : Correlation coefficient $f_{A B}^{(A)}$ in a BCC alloy dilute in $B$ as a function of the solute concentration $C(B)$. Dumbbells jump with the simple translation mechanism, the set of jump frequencies is indicated in the text. Full circles stand for Monte Carlo simulations by Sharma et al. [11] ; the dashed line refers to the model of Sharma et al., the dotted line to the first shell approximation of the SCMF theory and the full line to the second shell approximation of the SCMF theory.

Figure 4 : Transport coefficients in a BCC alloy dilute in $B$ as a function of the association frequency $w_{3}$; the frequencies $w_{0}, w_{1}$ and $w_{2}$ are equal to 1 , as well as all factors $\tau_{i}$, and the on-site rotation is discarded. Symbols stand for Monte Carlo simulations, dashed lines for the first shell approximation of the SCMF (equivalent to the expression of Barbu and Lidiard [10]), and full lines for the SCMF in second shell approximation.

Figure 5 : Energetic description of the stairs-like behaviour : the (negative) binding energy of the mixed dumbbell $\epsilon_{A B}$ is fixed and the solute-dumbbell binding energy $V_{A A_{\|} B}$ can vary from 0 to $\epsilon_{A B}$. All frequencies are equal to 1, excepted for the dissociation frequencies of the mixed dumbbell $w_{2}$ and of the solute-dumbbell complex $w_{4}$.

Figure 6 : Transport coefficients in a BCC alloy dilute in $B$ for the stairs-like behaviour described http://mc.manuscriptcentral.com/pm-pml 
in Figure 5, as a function of the frequency $w_{4}$. The binding energy of the mixed dumbbell is $\exp \left(-\beta \epsilon_{A B}\right)=100$. Dashed lines stand for the SCMF in first shell approximation, solid lines for the SCMF in second shell approximation, symbols for Monte Carlo simulations. 


1
2
3
4
5
6
7
8
9
10
11
12
13
14
15
16
17
18
19
20
21
22
23
24
25
26
27
28
29
30
31
32
33
34
35
36
37
38
39
40
41
42
43
44
45
46
47
48
49
50
51
52
53
54
55
56
50

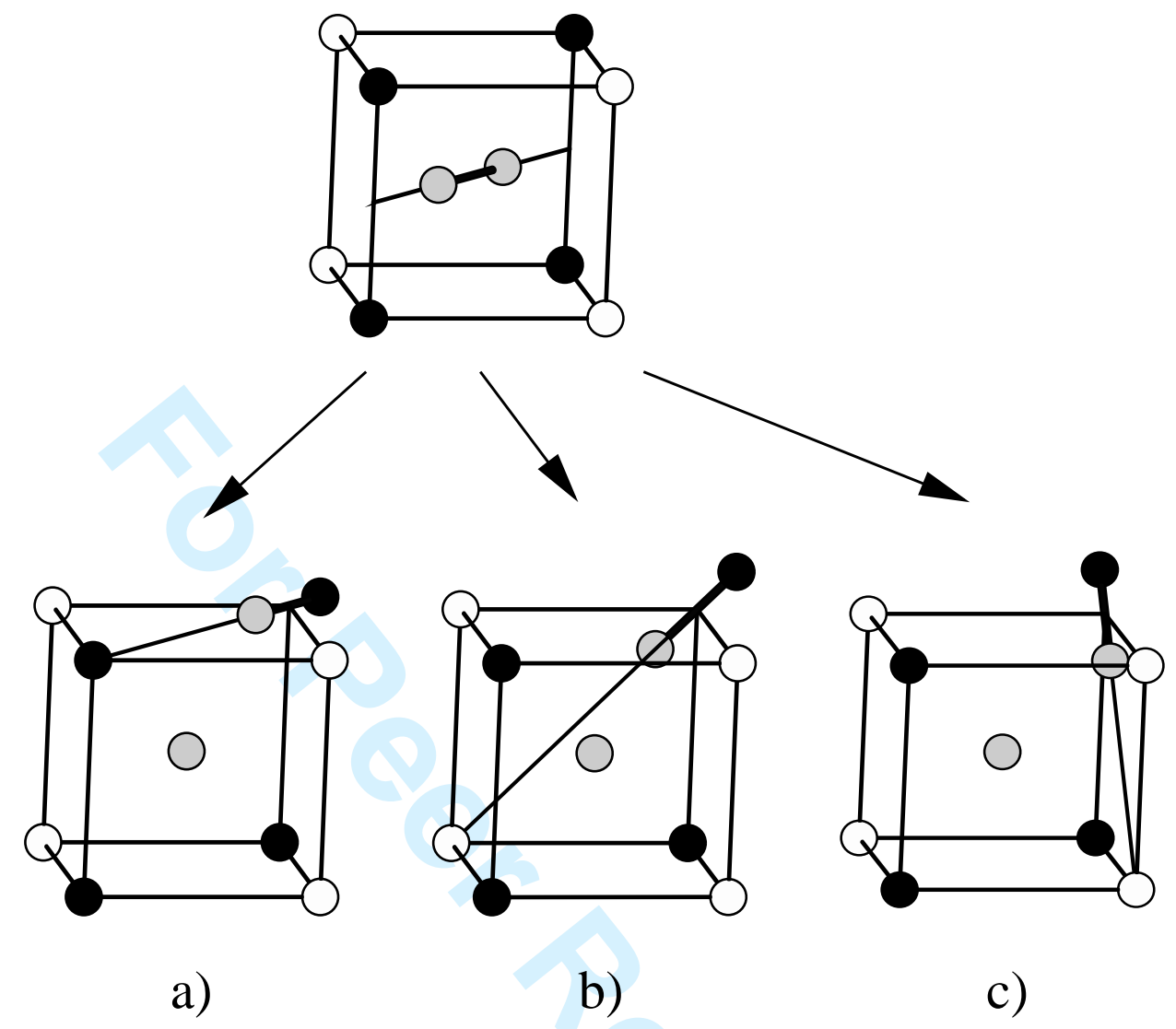

Figure 1: 
Philosophical Magazine \& Philosophical Magazine Letters

Page 32 of 42

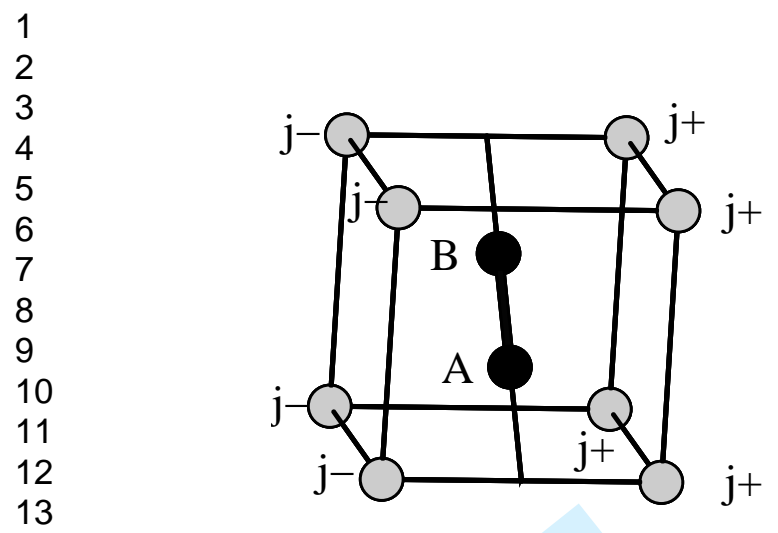

(a)

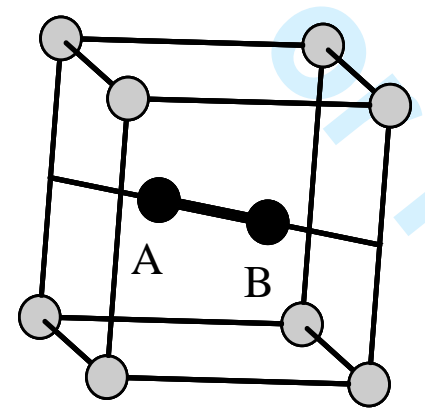

(d)

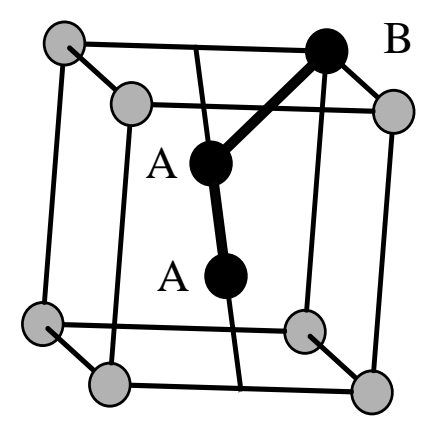

(b)

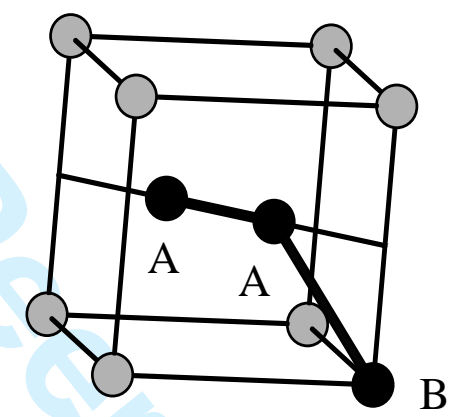

(e)

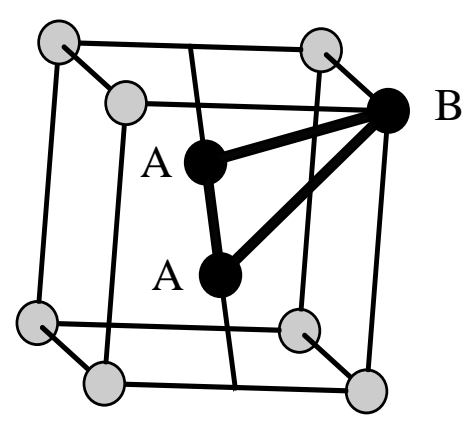

(c)

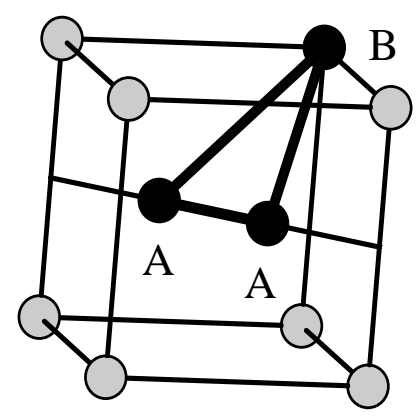

(f)

Figure 2:

http://mc.manuscriptcentral.com/pm-pml

32 


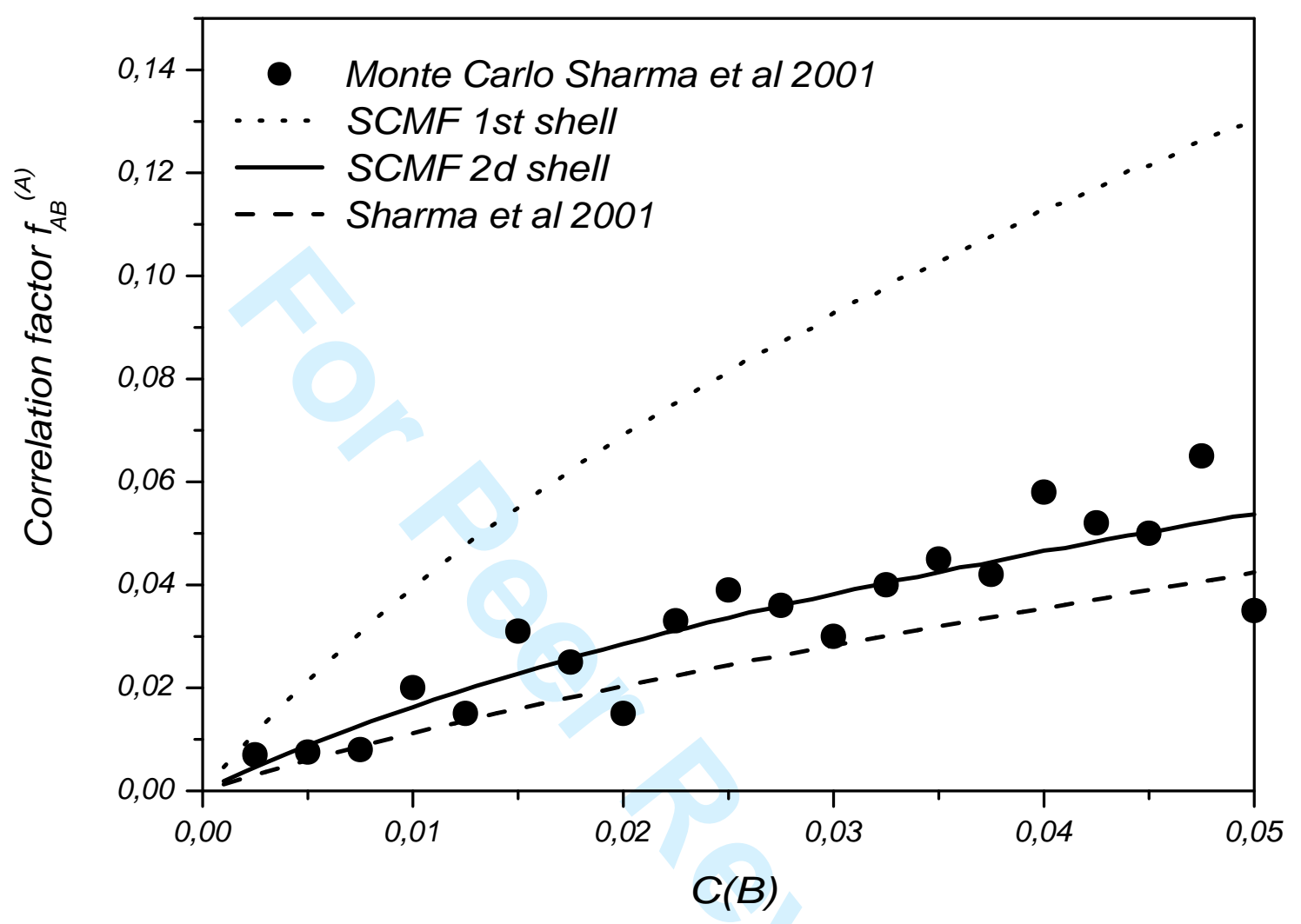

Figure 3:

http://mc.manuscriptcentral.com/pm-pml 


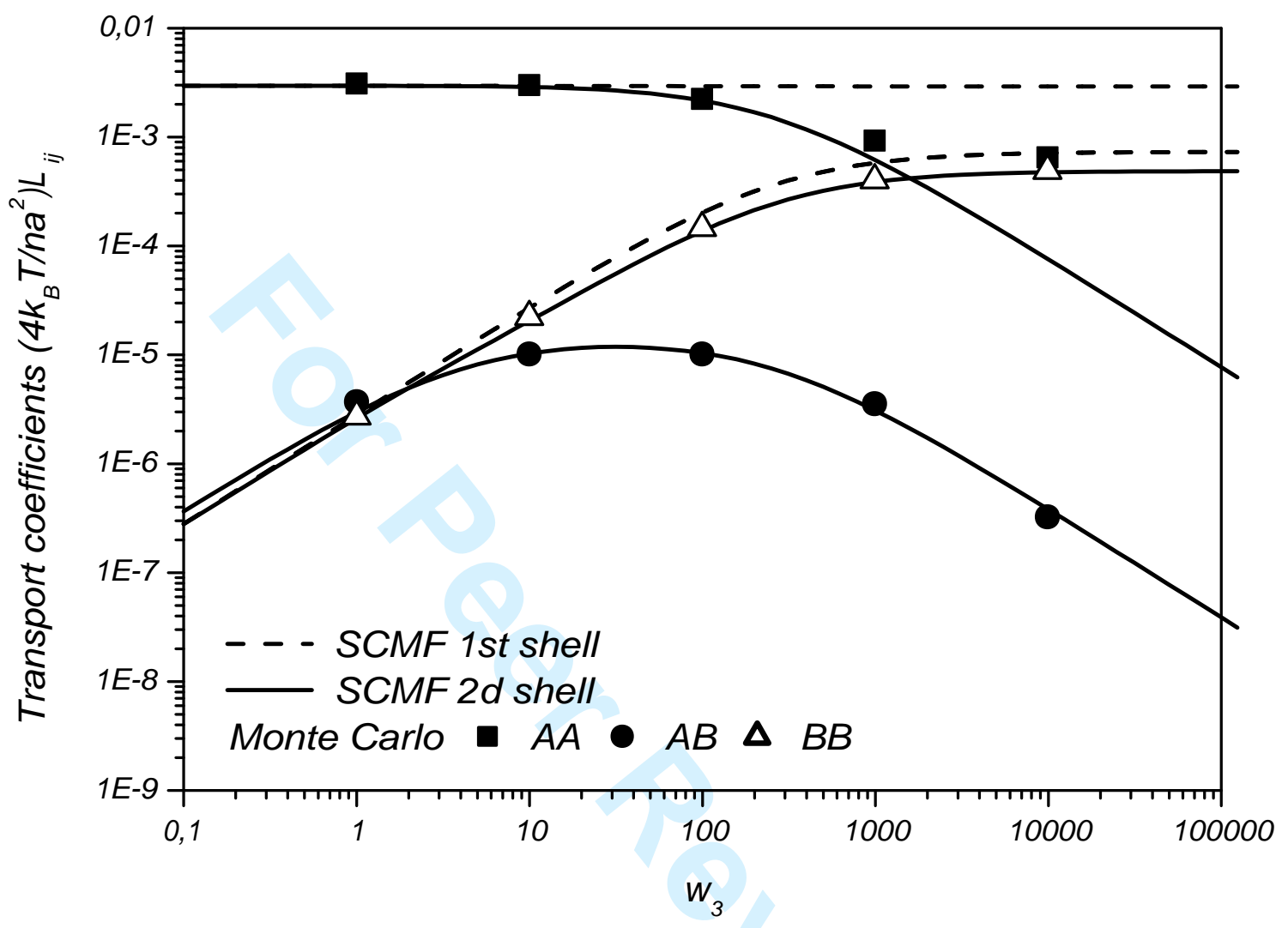

Figure 4:

http://mc.manuscriptcentral.com/pm-pml 


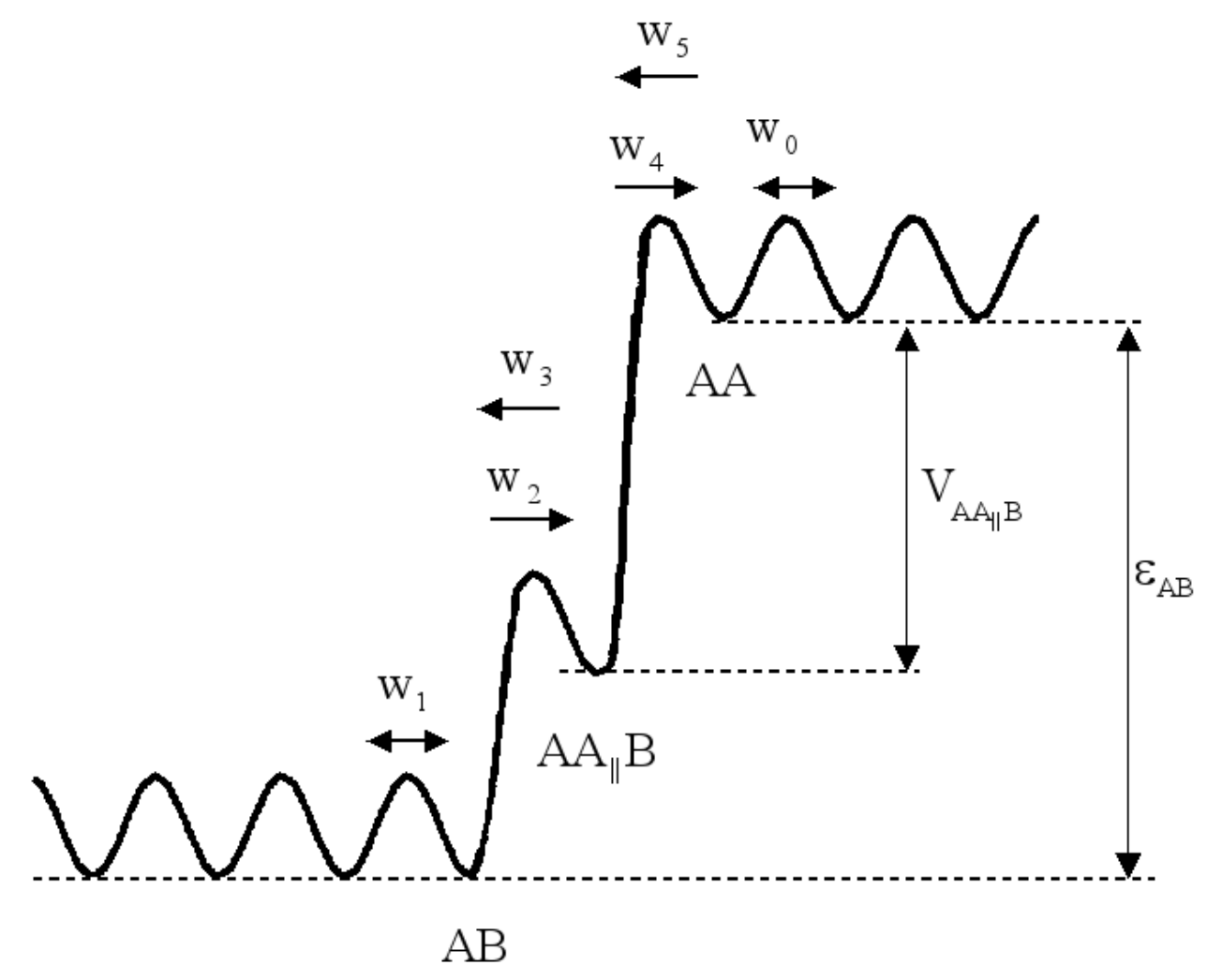

Figure 5:

http://mc.manuscriptcentral.com/pm-pml 


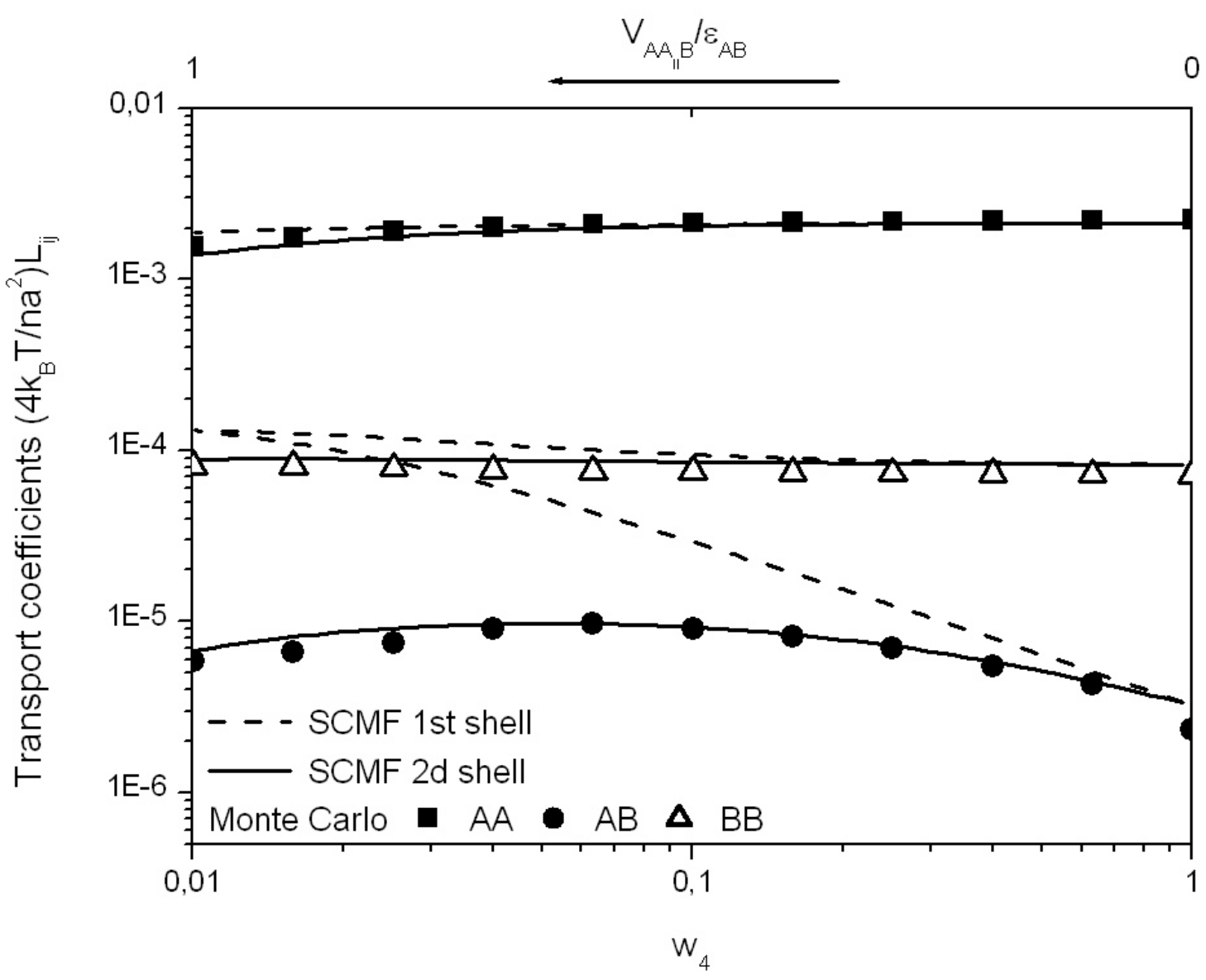

Figure 6:

http://mc.manuscriptcentral.com/pm-pml 
Page 37 of Prilosophibal Magazine \& F hilosophical Magazine Letters

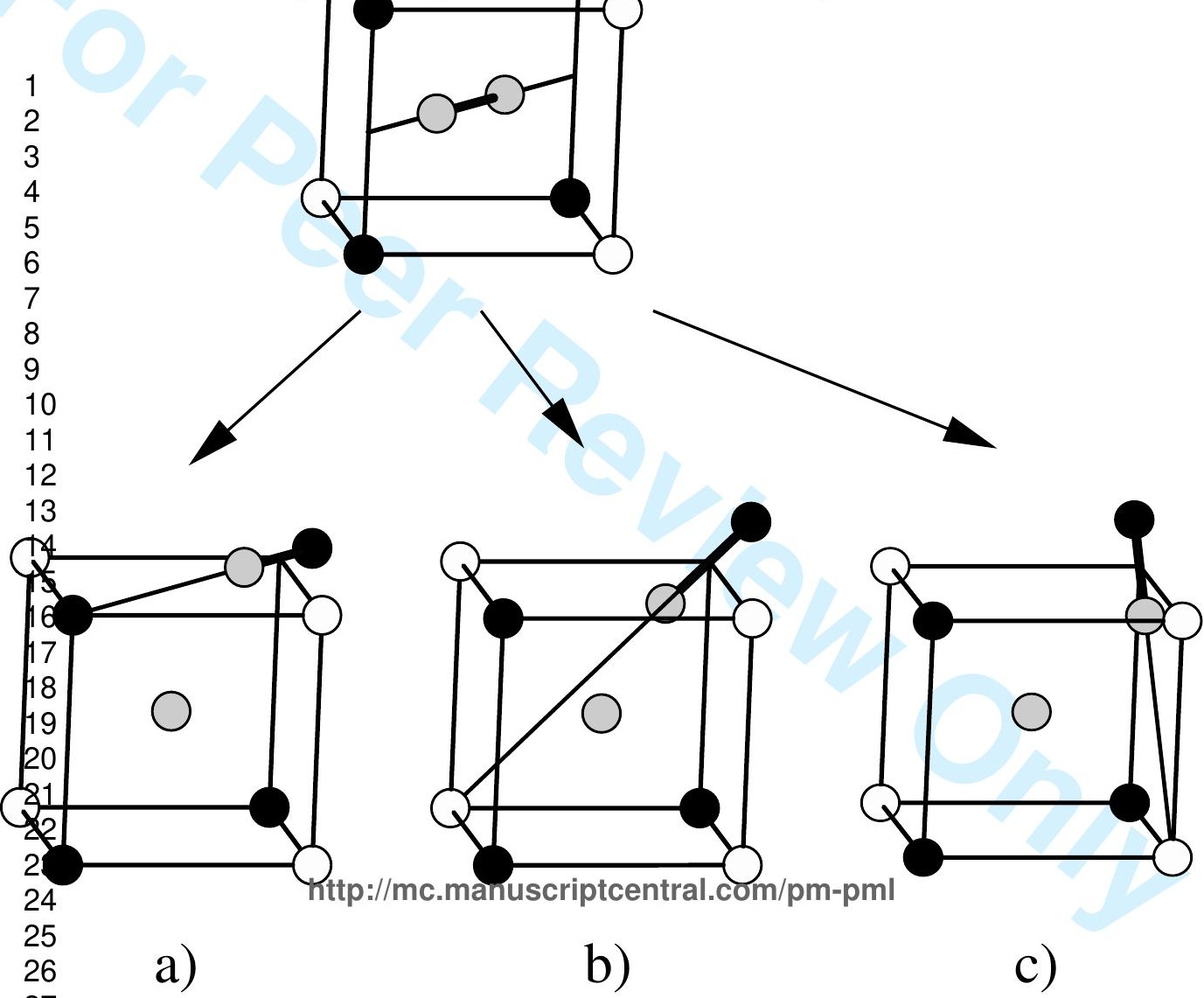




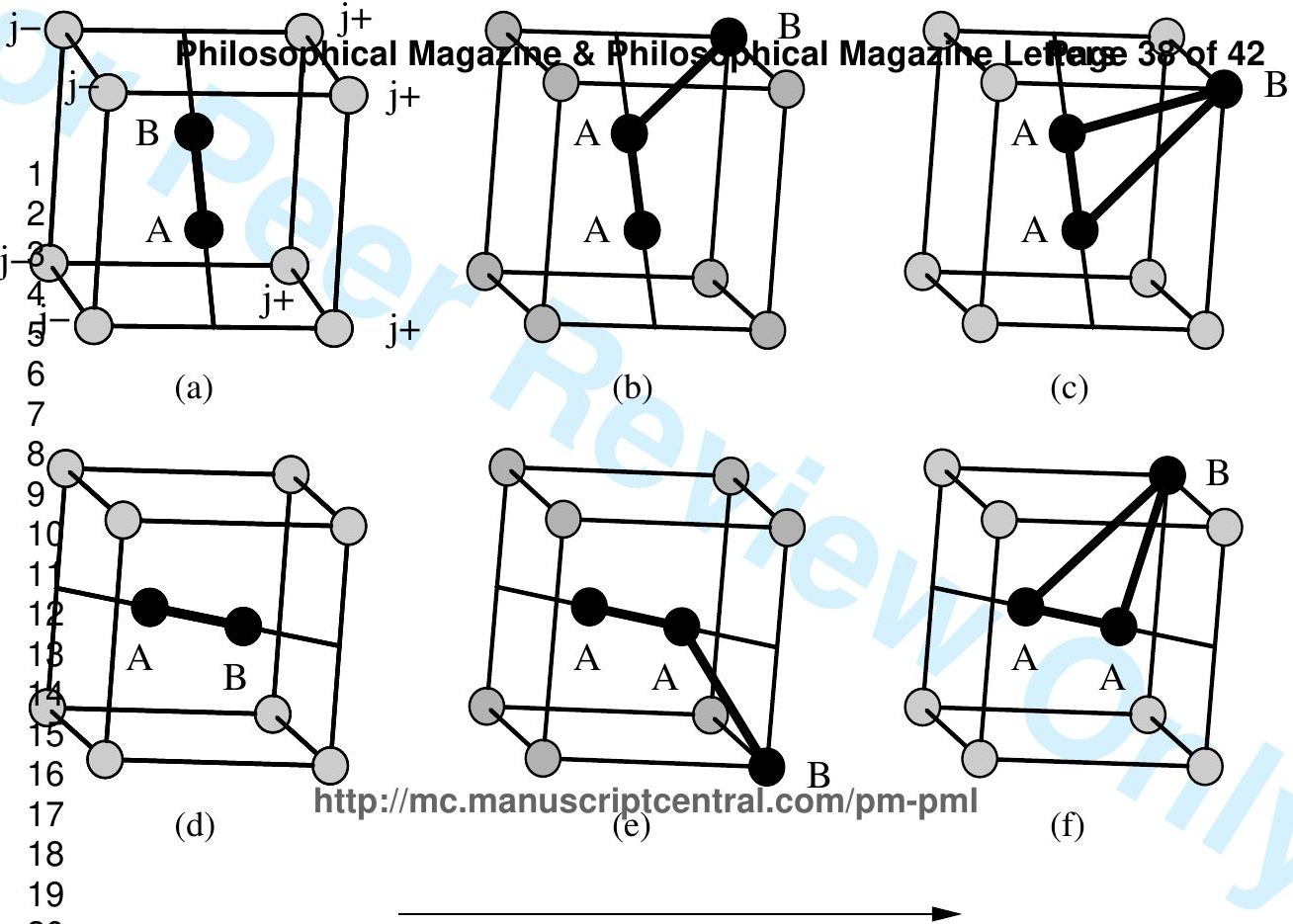




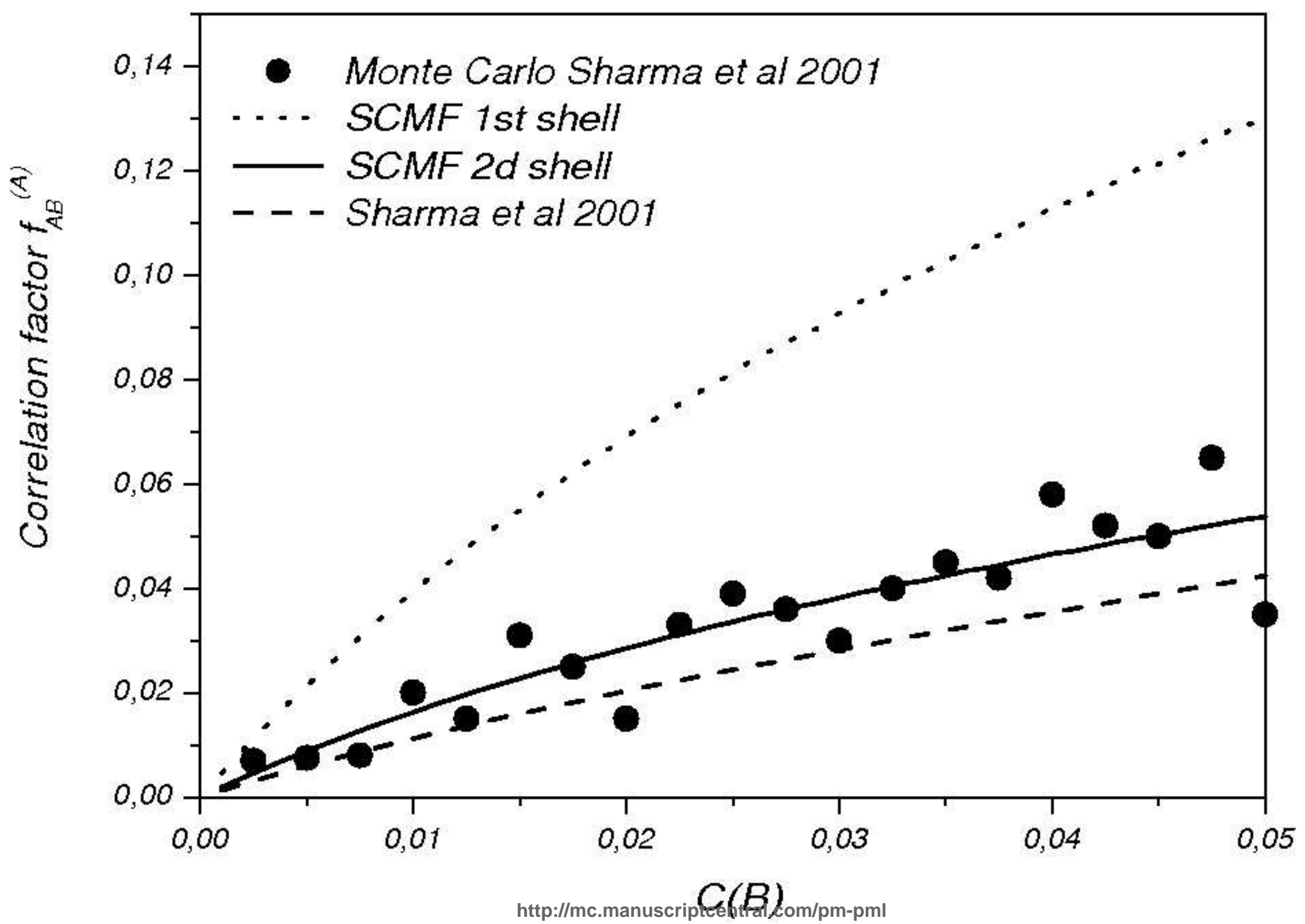




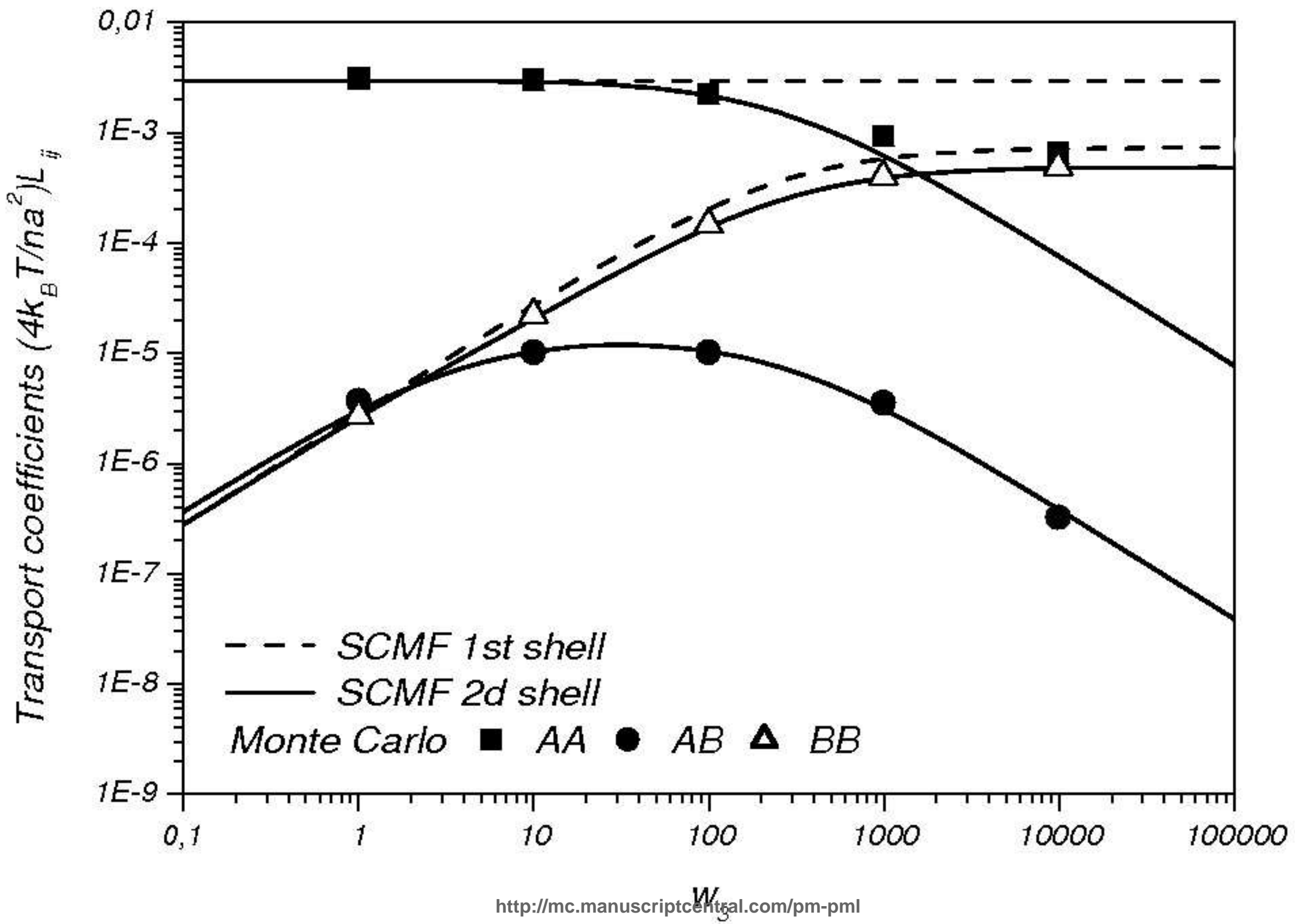




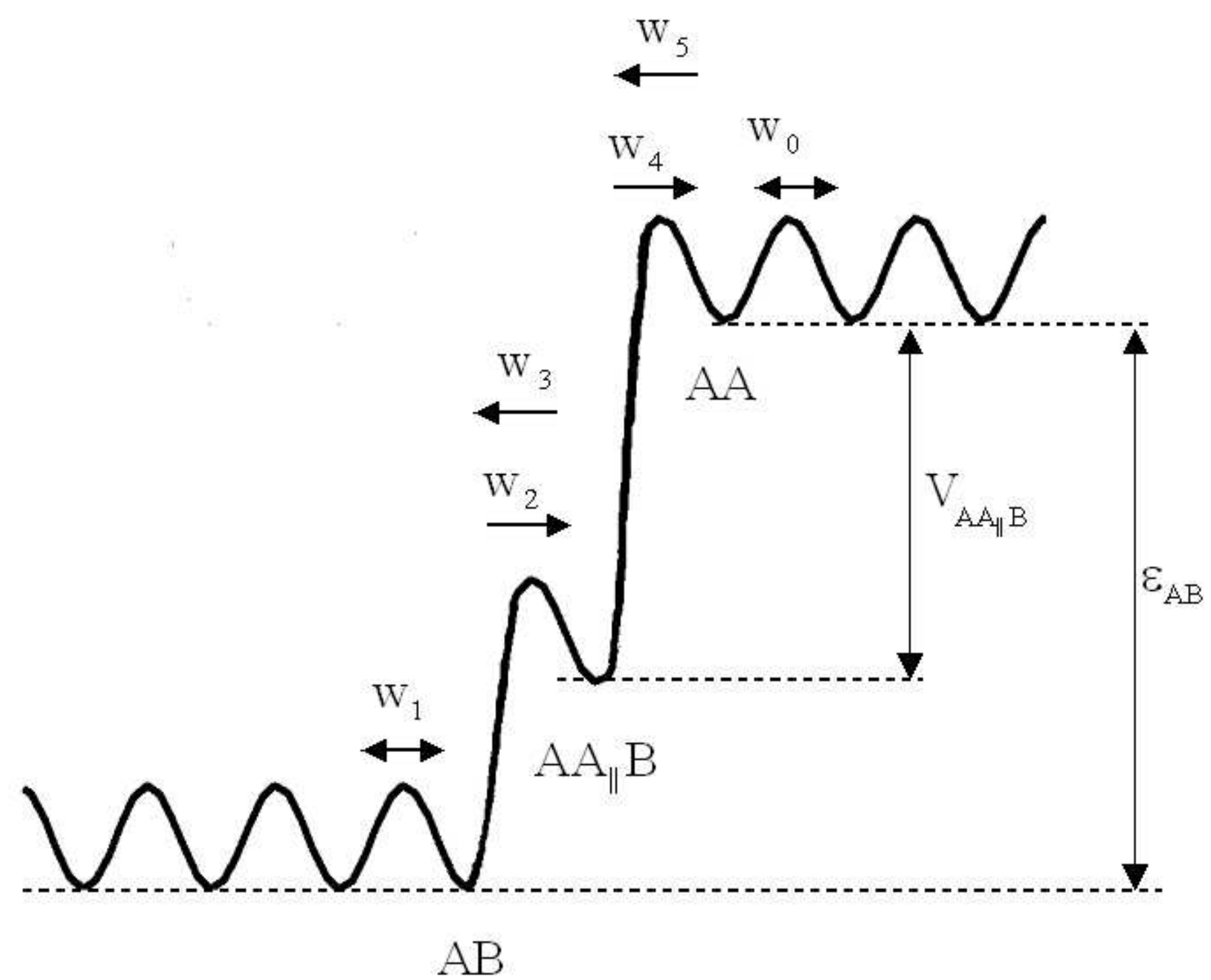

http://mc.manuscriptcentral.com/pm-pml 


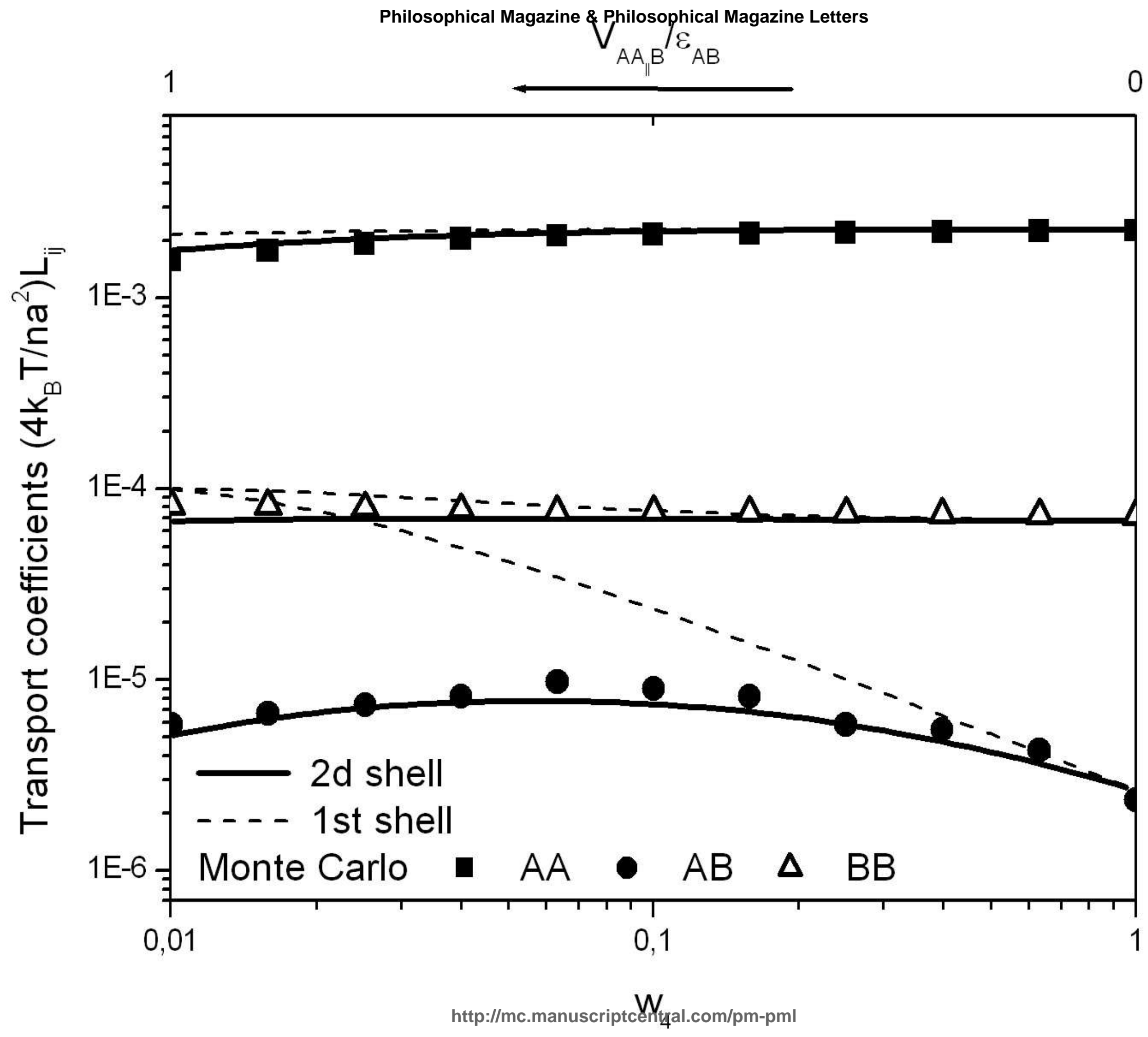

